\title{
Treatment discontinuation and clinical outcomes in the I-year naturalistic treatment of patients with schizophrenia at risk of treatment nonadherence
}

\author{
This article was published in the following Dove Press journal: \\ Patient Preference and Adherence \\ 6 May 2011 \\ Number of times this article has been viewed
}

\section{Katarina Kelin' \\ Timothy JR Lambert ${ }^{2}$ \\ Alan JM Brnabic ${ }^{3}$ \\ Richard Newton ${ }^{4}$ \\ Wendy $\mathrm{Ye}^{3}$ \\ Raúl I Escamilla ${ }^{5}$ \\ Kuang-Peng Chen ${ }^{6}$ \\ Liana Don ${ }^{7}$ \\ William Montgomery ${ }^{\prime}$ \\ Jamie Karagianis ${ }^{8}$ \\ Haya Ascher-Svanum ${ }^{9}$ \\ 'Eli Lilly Australia Pty Ltd, West Ryde, NSW, Australia; ${ }^{2}$ Discipline of Psychiatry, Brain and Mind Research Institute, The University of Sydney, Camperdown, NSW, Australia; \\ ${ }^{3}$ Intercontinental Information Sciences, Eli Lilly Australia Pty Ltd, Macquarie Park, NSW, Australia; ${ }^{4}$ Peninsula Health Psychiatric Services, Frankston Hospital, Frankston, VIC, Australia (current affiliation: Department of Psychiatry, Austin Hospital, Heidelberg, VIC, Australia); ${ }^{5}$ Schizophrenia Clinic, National Institute of Psychiatry, Mexico City, Mexico; ${ }^{6}$ Department of Psychiatry, National Taiwan University Hospital, Yun-Lin, Taiwan; ${ }^{7}$ Department of Psychiatry, University of Medicine Iuliu Hatieganu Cluj Napoca, Romania; ${ }^{8}$ Lilly USA, Eli Lilly and Company, Indianapolis, IN, USA; ' Global Health Outcomes, Lilly Research Laboratories, Eli Lilly and Company, Indianapolis, IN, USA}

Correspondence: Katarina Kelin Eli Lilly Australia Pty Ltd, I I 2 Wharf Road, West Ryde, NSW 2I I4, Australia

Tel +6I 293254672

Fax +6I 293254699

Email kelin_katarina@lilly.com
Background: This study aimed to improve physicians' understanding of the treatment circumstances and needs of outpatients with schizophrenia at risk of nonadherence, by naturalistically assessing antipsychotic treatment patterns, clinical outcomes, and health care service use in this little-studied patient population.

Methods: In this one-year, prospective, multicenter, noninterventional, observational study, patients considered at risk of nonadherence by their physicians were switched from their primary oral antipsychotic to another oral or a depot antipsychotic at study entry. All cause treatment discontinuation (antipsychotic switch, augmentation, or discontinuation) during the study was assessed using Kaplan-Meier survival analyses and descriptive statistics. Patients' illness severity, quality of life, attitude towards medication, patient-reported adherence, and health care resource utilization were assessed during the study.

Results: Of the 406 enrolled patients, 43 (10.6\%) were switched to depot and $363(89.4 \%)$ were switched to oral antipsychotics at study entry. During the study, 99 (24.4\%) patients switched, augmented, or discontinued their antipsychotic (all cause treatment discontinuation). Of the 99 patients who switched, augmented, or discontinued their antipsychotic, 8 (18.6\%) were taking depot and 91 (25.0\%) were taking oral antipsychotics. These patients were switched to either depot $(n=15)$ or oral $(n=78)$ antipsychotics, or discontinued their antipsychotic medication $(n=6)$. Inadequate response was the most frequently reported reason for medication discontinuation. During the study, patients' clinical and functional status improved significantly and service use was low. Most patients considered themselves to be adherent at study entry, and this favorable self-perception increased during the study (from $68.5 \%$ to $88.1 \%$ ).

Conclusion: Although identified as at risk of nonadherence, few patients in this naturalistic study discontinued their prescribed antipsychotic medication during the study. The discrepancy between the physicians' perception of their patient's medication adherence and the patients' self-perceived adherence highlights the need to better understand the underlying reasons for this phenomenon.

Keywords: antipsychotic drugs, medication persistence, outpatients, schizophrenia, depot antipsychotic, nonadherence

\section{Background}

Maintenance therapy with antipsychotic medication is necessary for the long-term clinical management of schizophrenia and is a key determinant for good prognosis. ${ }^{1}$ Patients who discontinue their antipsychotic medication regimen do so for many reasons, such as insufficient efficacy or tolerability, ${ }^{2,3}$ and are at increased risk of relapse and psychiatric hospitalization, ${ }^{4-6}$ symptom exacerbation, ${ }^{7}$ and poor 
functional outcomes. ${ }^{8,9}$ Maintenance therapy with antipsychotic medication is also compromised by patient nonadherence, a common challenge for physicians treating patients with schizophrenia in outpatient settings. At some point during their illness, up to $80 \%$ of patients will not fully adhere to their medication, ${ }^{10}$ and those who do not take their medication as prescribed are at an increased risk of relapse. ${ }^{11}$

Time to all cause treatment discontinuation has been used in recent studies as a clinically meaningful composite measure of treatment efficacy, tolerability, and safety as perceived by the physician and patient. ${ }^{3,12}$ Most studies assessing time to all cause treatment discontinuation found that the choice of antipsychotic medication influenced the time to and rates of treatment discontinuation. . $^{2,3,8,13-18}$ Another potential factor that may influence treatment discontinuation is a patient's inherent risk of nonadherence. Nonadherence to antipsychotic medication in the previous six months was found to be the best single predictor of future nonadherence. ${ }^{19}$ However, few studies have assessed whether patients with schizophrenia who have been identified as being at risk of nonadherence (ie, do not regularly take their medication) are at increased risk of discontinuing their medication.

To the authors' knowledge, this is the first study to prospectively assess antipsychotic medication discontinuation over 12 months in outpatients with schizophrenia who were considered to be at risk of nonadherence by their physician. Patients were considered to be at risk of nonadherence according to one of five reasons: 1) insufficient efficacy, ${ }^{3}$ ) concerns around tolerability of the medication, ${ }^{3}$ 3 ) history of medication nonadherence, ${ }^{19,20} 4$ ) poor insight into having a mental illness, ${ }^{20}$ or 5) negative attitude to their medication. ${ }^{20}$ As this patient population had not been previously studied, the authors aimed to gather naturalistic, observational data to improve physicians' understanding of this patient segment, their clinical circumstances, and their treatment needs.

The primary objective of this study was to assess the time to all cause treatment discontinuation (defined as any antipsychotic medication switch, augmentation, or discontinuation). The secondary aims of this study were to report the antipsychotic usage patterns; the reasons for antipsychotic discontinuation and the time to treatment discontinuation for each of the main reasons; the change in patients' illness severity, quality of life, and attitude towards their medication; and concomitant medication use, patient-reported adherence, and resource utilization.

\section{Methods}

\section{Study design}

This was a one-year, prospective, multicenter, noninterventional, observational study of outpatients with schizophrenia who required a change in their primary antipsychotic medication because of a physician-perceived risk of nonadherence. The study was conducted from April 2007 to July 2009 in 31 clinical practice sites located in Australia (10), Mexico (3), Romania (14), and Taiwan (4).

All patients provided voluntary, written informed consent for the use of their personal health information collected in the study. The study was conducted in accordance with the Declaration of Helsinki and the applicable laws and regulations of the study countries and regions, and was confirmed to be noninterventional by ethical review boards in each study country and region.

\section{Study population}

The study population has been described previously. ${ }^{21}$ In brief, male or female outpatients meeting diagnostic criteria for schizophrenia (DSM-IV or DSM-IV TR) ${ }^{22}$ were included in this study if they: 1) were aged between 18 and 65 years; 2) had at least two previous episodes of clinical worsening requiring hospitalization or increased level of care in the 24 months before study entry; 3 ) were considered at risk of nonadherence (lack of insight, negative drug attitude, documented history of medication nonadherence, inadequate response to the primary antipsychotic medication, or intolerance to the primary antipsychotic medication) by their physician; and 4) required a switch of their primary oral antipsychotic medication. Patients were excluded if they were considered by their physician to be treatment-resistant, or were receiving clozapine because of treatment resistance at study screening; were pregnant or nursing; or had an acute, serious, or unstable medical condition.

\section{Treatment}

All aspects of treatment and care of patients were determined by the treating physicians in keeping with their best clinical judgment. Treatment decisions were solely at the discretion of the physician and the patient, and treatment was prescribed according to the usual standard of care.

Enrolled patients were switched from their primary oral antipsychotic medication to another oral or a depot antipsychotic up to 90 days before study entry (Day 0). At the physician's discretion, the patient's antipsychotic prescribed at study entry could be switched, augmented, or discontinued during the study for any reason. Medication switches or 
augmentations included a switch to another antipsychotic, a change in antipsychotic formulation, or the addition of another antipsychotic. However, the addition of an oral antipsychotic to a depot antipsychotic or the discontinuation of an oral antipsychotic while taking a depot antipsychotic were not considered to be medication changes. Physicians selected reason(s) for any switch, augmentation, or discontinuation of antipsychotic from the following: poor insight, negative drug attitude, inadequate response, lack of compliance (related to a documented history of nonadherence), patient's request, investigator decision, clinical relapse, intolerance to drug, prescription inconvenience, social reasons, and financial cost of medication. Physicians' nominated the primary reason for any switch, augmentation, or discontinuation of antipsychotic if more than one reason was selected.

\section{Patient assessment}

Patients were observed for up to 12 months following enrollment, and were assessed at Day 0 (study entry), Month 3 (Visit 2), Month 12 (Visit 3), if they relapsed or withdrew from the study, and if they switched, augmented, or discontinued their antipsychotic between study visits. Patient sociodemographic characteristics were recorded at study entry, and patient-reported medication adherence and resource utilization (number of and reasons for hospital admissions, number of outpatient visits to any physician including psychiatrists and physicians other than psychiatrists, number of days in partial care, and number of emergency room or equivalent visits [overnight stay] for psychiatric illness and nonpsychiatric illness) were recorded at all visits. At study entry, patients also reported on their alcohol consumption, smoking, and illicit drug use for the six months prior to study entry. Detailed information regarding patient consumption habits and other sociodemographic characteristics have been published previously. ${ }^{21}$

Illness severity, assessed by the physician using the Clinical Global Impressions of Severity scale (CGI-S; 1 is not ill, 7 is extremely ill), ${ }^{23}$ and patient's attitude to their medication(s), assessed using the self-report, 10-item Drug Attitude Inventory questionnaire (DAI-10; -10 is negative attitude, 10 is positive attitude), ${ }^{24}$ were recorded at all visits. Quality of life was assessed using the self-report European Quality of Life instrument (EuroQoL EQ-5D) at study entry and Month $12,{ }^{25}$ whereas health-related quality of life was assessed using the patient-rated, 12-item Short-Form health survey (SF-12; 100 is best imaginable health state) at all visits. ${ }^{26}$ United Kingdom adult population-based preferences for the EQ-5D health states (100 is best imaginable health state) were used to calculate the EQ-5D utility score (1 is perfect health). ${ }^{27}$ The safety and tolerability data will be reported in a following publication of the study data.

\section{Statistical analysis}

All patients who provided consent to release information, and who fulfilled the study entry criteria were included in the analyses. The analyses included data, up to the point of last data collection, for patients who were lost to follow-up or who dropped out of the study. Descriptive statistics were used to calculate the mean and standard deviation (SD) or the median, minimum ( $\min$ ), and maximum ( $\max$ ) for continuous variables, and counts and percentages for categorical variables. The mean \pm SD dosage of each antipsychotic prescribed at study entry was calculated taking into account the days on therapy until treatment or study discontinuation. Statistical significance was prespecified at a two-sided $P<0.05$. Statistical Analysis Software (SAS; v 9.1.3, SAS Institute, Inc, Cary, NC) was used for all statistical analyses.

\section{Survival analysis}

Survival analyses were conducted for time to all cause treatment discontinuation. All cause treatment discontinuation was defined as any switch, augmentation, or discontinuation of antipsychotic medication for any reason. Patients who discontinued the study for any reason were assumed to be still taking their medication. Time to discontinuation was calculated from the date of study entry to the date of the first all cause treatment discontinuation. Survival curves were constructed using unadjusted Kaplan-Meier estimates. If appropriate, the median survival time estimates and 95\% confidence intervals (CI) are presented. For the survival analyses, patients were censored if they discontinued from study for any reason, eg, death, lost to follow-up, or patient decision. The analysis was truncated at 365 days so that all patients had exactly 1 -year of follow up.

\section{Change in clinical and functional status analyses}

Analyses of the CGI-S, DAI-10, and EQ-5D scores excluded patients who switched antipsychotics more than seven days before their first study visit and analyses of SF-12 scores excluded patients who switched antipsychotics more than 28 days before their first study visit to provide a more accurate representation of patient's clinical and functional illness profile at study entry. The temporal changes in CGI-S and SF-12 scores were analyzed using mixed model repeated measures (MMRM) and in EQ-5D scores were analyzed 
using analysis of covariance (ANCOVA). Both the MMRM and ANCOVA were controlled for sociodemographics (age, gender, income status, marital status, smoking status, work status, and alcohol consumption during the six months before study entry) and baseline clinical characteristics (age at first episode, number of previous episodes or exacerbations of schizophrenia in the previous 24 months, duration of current episode of schizophrenia, hospitalization in the six months before study entry, concomitant use of anticholinergics at study entry, concomitant use of benzodiazepines at study entry, previous use of antidepressants, previous use of anxiolytics, sedatives, or hypnotics, previous use of mood stabilizers, body mass index [BMI], CGI-S score, EQ-5D health state score, EQ-5D utility score, SF-12 mental health score, and SF-12 physical component score). Measures of significance were based on the least square (LS) mean difference and type III sum-of-squares using a two-sided $\alpha=0.05$. The unstructured covariance structure was prespecified to model the within-patient errors. Only visits that were completed within the acceptable time frame for the visit were included.

\section{Treatment response and relapse analyses}

Patients were classified as responders if their CGI-S score decreased by $\geq 2$ points from a score of $\geq 4$ (moderately to extremely ill) at study entry, or by $\geq 1$ point(s) from a score of 3 (mildly ill) at study entry. Patients with a CGI-S score of 1 or 2 were excluded from this analysis, and patients who responded and then relapsed were eligible to be classified as a responder after changing antipsychotic medication. Patients were classified as relapsed if they were classified as responders and their CGI-S score increased to that recorded at study entry or higher, and/or by $\geq 2$ points from the lowest CGI-S score recorded at the previous visit.

\section{Results}

\section{Patient disposition}

Figure 1 provides an overview of patient disposition at study entry, which has previously been reported in detail. ${ }^{21}$ Of the 440 patients considered to be at risk of nonadherence and who entered the study, 406 patients were enrolled (Australia, $n=64$; Mexico, $n=40$; Romania, $n=156$; Taiwan, $n=146$ ). The remaining 34 patients were excluded because of no data entry $(n=4)$, no antipsychotic change $(n=22)$, or an antipsychotic switch more than 90 days before study entry $(n=8)$.

Of the 406 enrolled patients, 318 (78.3\%) completed the study. Fewer patients in Australia (71.9\%, 46/64) and Taiwan $(63.0 \%, 92 / 146)$ completed the study than patients

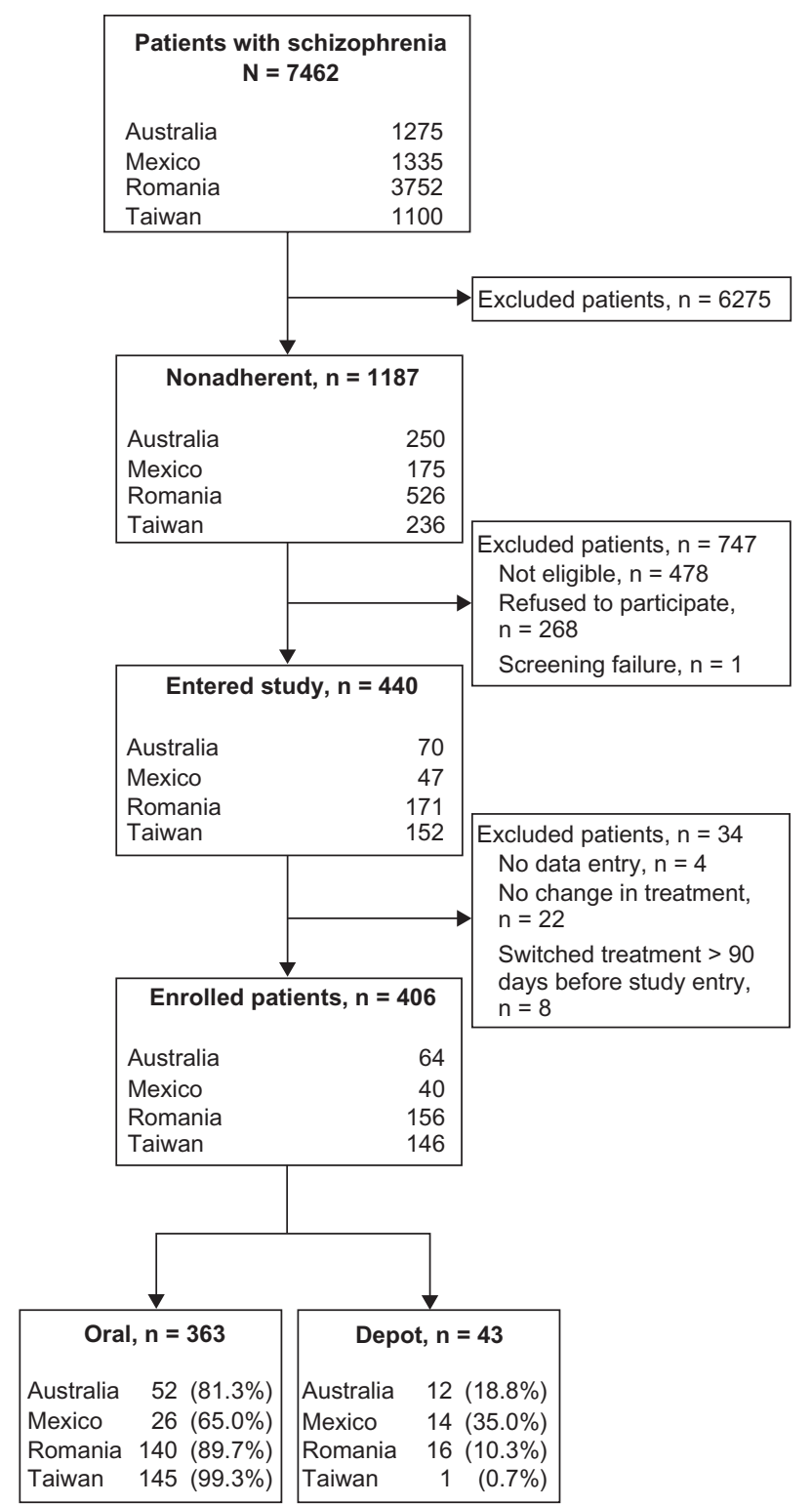

Figure I Patient disposition.

in Mexico (92.5\%, 37/40) and Romania (91.7\%, 143/156). Patients $(\mathrm{n}=88)$ who discontinued from the study did so because of: lost to follow up $(58.0 \%, 51 / 88)$, subject decision $(8.0 \%, 7 / 88)$, investigator decision $(1.1 \%, 1 / 88)$, parent/caregiver decision $(1.1 \%, 1 / 88)$, clinical relapse $(1.1 \%$, $1 / 88)$, or other reasons that were not reported $(30.7 \%$, 27/88).

\section{Patient sociodemographic characteristics}

Patient sociodemographic characteristics at study entry have been reported in detail. ${ }^{21}$ Patients were in their late $30 \mathrm{~s}$ (mean $\pm \mathrm{SD}, 37.2 \pm 10.2$ years), mostly male $(56.7 \%$, $230 / 406)$, and had a mean \pm SD BMI of $26.1 \pm 5.56 \mathrm{~kg} / \mathrm{m}^{2}$. At study entry, most patients reported that they had never 
married or had no previous or current relationship $(59.6 \%, 242 / 406)$, lived with their family $(82.0 \%, 333 / 406)$, were unemployed $(43.4 \%, 176 / 406)$, and had no income (including pensions and allowances; 54.6\%, 223/406). In the six months before study entry, 25.9\% (105/406) of patients reported they had consumed alcohol and $6.2 \%$ $(25 / 406)$ reported they had taken illicit drugs.

\section{Reasons for risk of nonadherence at study entry}

Of the 406 enrolled patients, 224 (55.2\%) were considered to be at risk of nonadherence by their physician because of an inadequate response to the primary antipsychotic medication. Of the remaining patients, 73 (18.0\%) were considered at risk of nonadherence because of a negative drug attitude, $71(17.5 \%)$ because of intolerance to the primary antipsychotic medication, $31(7.6 \%)$ because of a documented history of medication nonadherence, and $7(1.7 \%)$ because of a lack of insight.

\section{Antipsychotic switch patterns at study entry}

Patients were switched from their primary oral antipsychotic medication to another oral antipsychotic $(89.4 \%, 363 / 406)$ or a depot antipsychotic $(10.6 \%, 43 / 406)$ within 90 days of study entry (Table 1). Most patients (84.5\%, 343/406) had switched antipsychotics within seven days of study entry. Patients switched to depot antipsychotics were switched to either first-generation $(48.8 \%, 21 / 43)$ or second-generation $(51.2 \%, 22 / 43)$ depot antipsychotics. Zuclopenthixol decanoate $(n=8)$, haloperidol decanoate $(n=5)$, and pipotiazine palmitate $(n=5)$ were the most frequently prescribed firstgeneration depot antipsychotics, whereas risperidone longacting injection $(n=22)$ was the only prescribed second-generation depot antipsychotic. The mean \pm SD

Table I Antipsychotic switch patterns at study entry

\begin{tabular}{ll}
\hline Antipsychotic class & $\mathbf{n}(\%)$ \\
\hline Depot & $\mathbf{4 0 ( 9 . 9 )}$ \\
First-generation & $20(4.9)$ \\
Second-generation & $20(4.9)$ \\
Depot + oral & $\mathbf{3}(\mathbf{0 . 7})$ \\
First-generation + first-generation & $\mathrm{I}(0.2)$ \\
Second-generation + second-generation & $2(0.5)$ \\
Oral & $\mathbf{3 6 2 ( 8 9 . 2 )}$ \\
First-generation & $38(9.4)$ \\
Second-generation & $324(79.8)$ \\
Oral + oral & $\mathbf{I}(\mathbf{0 . 2})$ \\
Second-generation + first-generation & $\mathrm{I}(0.2)$ \\
\hline
\end{tabular}

dosage of these antipsychotics was $314.1 \pm 217.4 \mathrm{mg} / \mathrm{month}$ for zuclopenthixol, $73.4 \pm 25.3 \mathrm{mg} / \mathrm{month}$ for haloperidol, $92.0 \pm 34.6 \mathrm{mg} / \mathrm{month}$ for pipotiazine, and $72.6 \pm 25.7 \mathrm{mg} / \mathrm{month}$ for risperidone. Patients switched to oral antipsychotics were mostly switched to second-generation $(89.5 \%, 325 / 363)$ rather than first-generation $(10.5 \%, 38 / 363)$ agents. Olanzapine $(\mathrm{n}=93)$, amisulpride $(\mathrm{n}=55)$, risperidone $(n=48)$, and aripiprazole $(n=47)$ were the most frequently prescribed second-generation oral antipsychotics, and haloperidol $(n=10)$ and trifluoperazine $(n=10)$ were the most frequently prescribed first-generation oral antipsychotics. The mean \pm SD dosage of these antipsychotics was $13.6 \pm 8.39 \mathrm{mg} /$ day for olanzapine, $609.1 \pm 536.4 \mathrm{mg} /$ day for amisulpride, $15.81 \pm 9.67 \mathrm{mg} /$ day for aripiprazole, $3.89 \pm 2.41 \mathrm{mg} /$ day for risperidone, $5.54 \pm 2.21 \mathrm{mg} /$ day for haloperidol, and $9.65 \pm 4.86 \mathrm{mg} /$ day for trifluoperazine.

\section{Time to treatment discontinuation}

Of the 406 patients enrolled in the study, 99 (24.4\%) switched, augmented, or discontinued their antipsychotic medication during the 12-month study period (all cause treatment discontinuation). As the proportion of patients who switched, augmented, or discontinued their medication did not reach $50 \%$ during the study, the median survival time for all cause treatment discontinuation could not be estimated from the Kaplan-Meier survival analysis (Figure 2). However, the 25th percentile (95\% CI) Kaplan-Meier estimate could be estimated at 310 (208, not applicable) days for all cause treatment discontinuation.

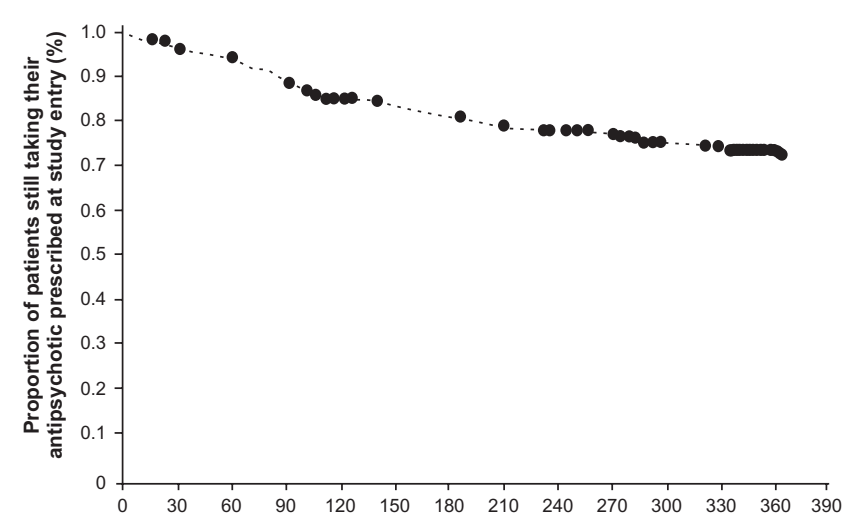

Figure 2 Twelve-month Kaplan-Meier survival curve $(n=406)$ for all cause treatment discontinuation (event, $\mathrm{n}=99$; censored, $\mathrm{n}=307$ ). Time to discontinuation was calculated from the date of study entry to the date of the first all cause treatment discontinuation. For the survival analyses, patients were censored if they had not switched, augmented, or discontinued their antipsychotic medication at 365 days, if they had completed the study, or if they discontinued from the study for any reason. Censored patients are denoted by $\bullet$. Median Kaplan-Meier estimates could not be calculated because the proportion of patients who had changed their medication during the study did not reach $50 \%$ at 12 months. 
In lieu of median Kaplan-Meier estimates, the median time to treatment discontinuation was summarized using descriptive statistics as $102(2,365)$ days $(n=99)$. Inadequate response $(35.9 \%)$ was the most frequently reported reason for treatment discontinuation (Table 2). Other frequently reported reasons were lack of compliance (14.1\%), investigator decision (13.0\%), and upon patient's request $(10.9 \%)$.

\section{Antipsychotic medication use patterns}

During the study, $8(18.6 \%)$ patients in the depot subgroup and $91(25.0 \%)$ patients in the oral subgroup switched, augmented, or discontinued their medication at least once (all cause treatment discontinuation, Table 3). Most of these patients $(93.9 \%, 93 / 99)$ switched or augmented their medication; 15 patients were switched to depot antipsychotics and 78 patients were switched to oral antipsychotics. Most patients switched to depot antipsychotics were previously taking an oral antipsychotic $(80.0 \%, 12 / 15)$. The remaining six patients, who were all taking oral antipsychotics, discontinued their medication.

A small number of patients had more than one antipsychotic medication switch or augmentation during the study. A further 26 patients (oral, $n=22$; depot, $n=4$ ) required a second switch or augmentation of their medication, and 3 patients (oral, $\mathrm{n}=3$ ) discontinued their medication after one previous switch in their medication. In addition, 8 patients (oral, $\mathrm{n}=7$; depot, $\mathrm{n}=1$ ) required a third, 2 patients (oral, $\mathrm{n}=2$ ) required a fourth, and 2 patients (oral, $\mathrm{n}=2$ ) required a fifth switch or augmentation of their medication. When study discontinuation was considered in addition to all cause treatment discontinuation, 66 patients (oral, $n=60$; depot, $n=6$ ) had not switched, augmented, or discontinued

Table 2 Reasons for all cause treatment discontinuation

\begin{tabular}{lll}
\hline Primary reason & $\mathbf{n}(\%)^{\mathbf{a}}$ & $\begin{array}{l}\text { Time (days) to treatment } \\
\text { discontinuation for each } \\
\text { reason, median (min, max) }\end{array}$ \\
\hline Negative drug attitude & $8(8.7)$ & $\mathrm{NC}$ \\
Inadequate response & $33(35.9)$ & $107(2,343)$ \\
Lack of compliance & $13(14.1)$ & $135(7,364)$ \\
Upon patients' request & $10(10.9)$ & $101.5(7,200)$ \\
Investigator decision & $12(13.0)$ & $91.5(8,300)$ \\
Clinical relapse & $7(7.6)$ & $\mathrm{NC}$ \\
Intolerance to drug & $9(9.8)$ & $\mathrm{NC}$
\end{tabular}

Notes: ${ }^{2}$ For the calculation of percentages, the denominator was $n=92$; ${ }^{\text {'Time to }}$ treatment discontinuation for each primary reason was estimated using data from patients who switched, augmented, or discontinued their antipsychotic medication for that reason only. Time to treatment discontinuation was not estimated for reasons with event numbers $<10$.

Abbreviations: max, maximum; min, minimum; NC, not calculated. their medication before discontinuing the study, 8 patients (oral, $\mathrm{n}=8$ ) had switched, augmented, or discontinued their medication once before discontinuing the study, and 1 patient (oral, $\mathrm{n}=1$ ) had switched, augmented, or discontinued his/her medication twice before discontinuing the study.

\section{Treatment response and relapse}

Of those patients who had a CGI-S score $>2$ at study entry, $22.5 \%(72 / 320)$ had responded to treatment at 3 months and $41.5 \%$ (132/318) had responded at 12 months. However, $13.7 \%(10 / 73)$ of responders had relapsed by 12 months.

\section{Change in clinical and functional status}

Statistically significant changes in the overall patient population's CGI-S, DAI-10, EQ-5D, and SF-12 scores from study entry to 12 months or endpoint were detected (Table 4). On average, patients' illness severity (CGI-S score) was classified as moderately ill at study entry and mildly ill by 12 months (MMRM of the LS mean change, $P<0.001$ ). Patients' attitude to their antipsychotic medication (DAI-10 score) changed from slightly positive at study entry and slightly more positive by 12 months or endpoint. In addition, patients rated their quality of life and level of functioning (EQ-5D and SF-12 scores) as low at study entry and higher by 12 months or endpoint (EQ-5D health state and utility scores, ANCOVA LS mean change, $P<0.001$; SF-12 mental health and physical component scores, MMRM analysis LS mean change, $P<0.001)$.

\section{Concomitant medication use}

Most patients (78.9\%, 311/394) took concomitant medication for schizophrenia during the study. Benzodiazepines or anticholinergics $(58.0 \%, 235 / 405)$ were the most common concomitant medication taken, followed by anxiolytics, sedatives, or hypnotics $(32.4 \%, 117 / 362)$, mood stabilizers (28.7\%, 104/362), and antidepressants (27.1\%, 97/358). In addition, most patients $(77.9 \%, 307 / 394)$ took other psychiatric medications, whereas few patients $(13.1 \%, 46 / 352)$ had received nonpharmacological interventions during the study.

\section{Patient-reported adherence}

The percentage of patients who considered themselves to be adherent increased during the study. At study entry, $68.5 \%(278 / 406)$ of patients considered themselves to have been adherent (they took all their medication or only missed taking their medication a couple of times) in the previous four weeks. In contrast, $86.8 \%(335 / 386)$ of patients at 
Table 3 Antipsychotic medication use patterns for all cause treatment discontinuation

\begin{tabular}{llll}
\hline & $\begin{array}{l}\text { Switch, augmentation, } \\
\text { or discontinuation, } \mathbf{n}(\%)\end{array}$ & $\begin{array}{l}\text { Switch or augmentation } \\
\text { only, } \mathbf{n} \text { (\%) }\end{array}$ & $\begin{array}{l}\text { Discontinuation } \\
\text { only, } \mathbf{n}(\%)\end{array}$ \\
\hline Overall $(\mathrm{n}=406)$ & $99(24.4)$ & $93(22.9)$ & $6(1.5)$ \\
Oral $(\mathrm{n}=363)$ & $91(25.1)$ & $85(23.4)$ & $6(1.7)$ \\
First-generation $(\mathrm{n}=38)$ & $15(39.5)$ & $15(39.5)$ & $0(0.0)$ \\
Second-generation $(\mathrm{n}=325)$ & $76(23.4)$ & $70(21.5)$ & $6(1.9)$ \\
Depot $(\mathrm{n}=43)$ & $8(18.6)$ & $8(18.6)$ & $0(0.0)$ \\
First-generation $(\mathrm{n}=21)$ & $3(14.3)$ & $3(14.3)$ & $0(0.0)$ \\
Second-generation $(\mathrm{n}=22)$ & $5(22.7)$ & $5(23.4)$ & $0(0.0)$ \\
\hline
\end{tabular}

3 months and $88.1 \%(304 / 345)$ of patients at 12 months considered themselves to have been adherent since their last study visit.

\section{Resource utilization}

During the study, $56(16.7 \%)$ patients had at least one psychiatric hospitalization. Of these patients, 39 (69.6\%) had one, 10 had two (17.9\%), and 7 (12.5\%) had three hospital admissions. Of the remaining two patients, one had four hospital admissions and the other had seven admissions. The most common reason for admission during the study was clinical relapse $(n=47[14.0 \%])$, followed by psychiatric condition other than schizophrenia $(n=6[1.8 \%])$, medical condition $(n=5[1.5 \%])$, and other reasons $(n=4[1.2 \%])$. In terms of the use of other resources, the frequency of outpatient visits to any physician, including psychiatrists, was lower during the study than during the 12 months before study entry. Fewer patients had eleven or more outpatient visits during the study (Month 3: $\mathrm{n}=23$ [6.2\%]; Month 12: $\mathrm{n}=52[15.8 \%])$ than before study entry $(\mathrm{n}=177[48.0 \%])$, whereas more patients had one to ten outpatient visits during the study (Month 3: $\mathrm{n}=317$ [85.7\%]; Month 12: $\mathrm{n}=265$ $[80.3 \%])$ than before study entry $(n=179[48.5 \%])$. Less than
$10 \%$ of patients had visited an emergency room or an equivalent facility (overnight stay) for psychiatric illness during the study, compared with about $45 \%$ of patients during the 12 months before study entry. The proportions of patients who had an outpatient visit to physicians other than psychiatrists, days in partial care, or an emergency room or equivalent facility visit (overnight stay) for nonpsychiatric illness during the study was less than those during the 12 months before study entry.

\section{Discussion}

This is the first study to assess all cause treatment discontinuation among outpatients with schizophrenia who were perceived by their physicians to be at risk of nonadherence to their antipsychotic medication regimen. Conducted in usual care settings using a prospective observational design, patients switched antipsychotics at study entry and most were switched to oral rather than depot antipsychotics. The primary analysis indicated that during the study, few patients $(24.4 \%)$ switched, augmented, or discontinued their antipsychotic medication prescribed at study entry. In addition, patients' clinical and functional illness status improved significantly and rates of psychiatric hospitalization and emergency

Table 4 Change in illness severity, attitude to antipsychotic medication, and quality of life scores

\begin{tabular}{|c|c|c|c|c|c|c|}
\hline \multirow[t]{2}{*}{ Score } & \multicolumn{2}{|c|}{ Study entry } & \multicolumn{4}{|c|}{ Month I 2} \\
\hline & $\mathbf{n}$ & Mean (SD) & $\mathbf{n}$ & Mean (SD) & LS mean change $(95 \% \mathrm{CI})$ & $P^{a}$ \\
\hline CGI-S & 343 & $4.18(0.97)$ & 291 & $3.23(1.08)$ & $-1.04(-1.24,-0.84)$ & $<0.001$ \\
\hline DAl-10 & 334 & $2.70(5.37)$ & 289 & $4.91(5.24)$ & $2.01(1.36,2.66)$ & $<0.001$ \\
\hline \multicolumn{7}{|l|}{ EQ-5D } \\
\hline Health state & 337 & $56.4(24.8)$ & 291 & $66.3(26.0)$ & $12.64(7.66,17.62)$ & $<0.001$ \\
\hline Utility ${ }^{f}$ & 324 & $0.62(0.30)$ & 277 & $0.77(0.25)$ & $0.16(0.10,0.23)$ & $<0.001$ \\
\hline \multicolumn{7}{|l|}{ SF-I $2^{c}$} \\
\hline Mental health & 391 & $35.2(10.9)$ & 332 & $4 I .5(11.4)$ & $7.09(5.11,9.06)$ & $<0.001$ \\
\hline Physical component & 391 & $44.2(9.20)$ & 332 & $47.4(8.97)$ & $4.52(2.88,6.15)$ & $<0.001$ \\
\hline
\end{tabular}

Notes: aChange in score from study entry to Month 12 (mixed-effect model for repeated measures analysis for CGI-S and SF-I2 scores; analysis of covariance for EQ-5D scores); ' Excluded patients who started treatment more than 7 days before their first study visit (patients eligible for assessment, $\mathrm{n}=343$ ); ${ }^{\mathrm{C}}$ Excluded patients who started treatment more than 28 days before their first study visit (patients eligible for assessment, $n=392$ ).

Abbreviations: CGI-S, Clinical Global Impressions of Severity; Cl, confidence interval; DAI- I0, I0-item Drug Attitude Inventory; EQ-5D, European Quality of life instrument; LS, least square; NC, not calculated; SD, standard deviation; SF-12, 12-item Short Form health survey. 
psychiatric service use were low during the study. Most patients considered themselves to be adherent to their medication at study entry, and this favorable self-perception increased during this 1-year study, showing a discrepancy between the physicians' perceptions of the patients' risk of nonadherence and the patient's assessment of their own adherence behaviors.

That few patients switched, augmented, or discontinued the antipsychotic medication prescribed at study entry was somewhat surprising given that the physicians considered these patients to be at risk of nonadherence. Even when study discontinuation was included, more than half of the patient group (59.4\%) had continued to take their initial therapy for at least one year. However, it is difficult to compare the current findings with previous research as most previous naturalistic, observational studies in this field have shown wide variations in all cause treatment discontinuation rates, depending on the specific antipsychotic prescribed. . $^{14,17,18,28}$ Although patients in previous studies were not assessed for their risk of nonadherence at study entry, the proportion of patients who completed one year of treatment without changing their antipsychotic medication varied from $22.7 \%$ to $71.6 \%$ in one large naturalistic United States (US) study, ${ }^{17}$ $53.7 \%$ to $69.1 \%$ in a prospective, observational Canadian study, ${ }^{28} 17.8 \%$ to $44.4 \%$ in the Intercontinental Schizophrenia Outpatient Health Outcomes (IC-SOHO) study, ${ }^{18}$ and 20.5\% to $48.6 \%$ in the European SOHO study. ${ }^{14}$

The low treatment discontinuation rate found in the current study may reflect the fact that more than $75 \%$ of patients were switched to a second-generation oral antipsychotic at study entry. This finding possibly reflects physicians' perceptions that these antipsychotics may provide better efficacy for patients with insufficient response. ${ }^{29}$ Although this study was not designed to assess treatment discontinuation rates for the individual antipsychotics, the 1-year treatment discontinuation rates in the current study are somewhat similar to those reported for olanzapine-treated patients in other naturalistic schizophrenia studies, eg, $24.4 \%$ versus $17.8 \%$ for IC-SOHO, ${ }^{18}$ and $23.0 \%$ for European SOHO, ${ }^{14}$ and when study discontinuation was considered, $40.6 \%$ versus $43.5 \%$ for the large naturalistic US study. ${ }^{17}$ However, in the current study, patients were switched to a variety of antipsychotics at study entry, in keeping with clinical practice, and only $22.9 \%$ of patients were switched to olanzapine thus rendering this potential explanation incomplete.

Although previous findings suggest that patients are less likely to discontinue depot antipsychotics than oral antipsychotics, ${ }^{30,31}$ it is unlikely that the prescription of depot antipsychotics at study entry influenced the treatment discontinuation rate in the current study. Few patients were switched to depot antipsychotics at study entry, despite being considered at risk of nonadherence. A number of factors may have influenced this result, including patient acceptance of, ${ }^{32}$ and preference for ${ }^{33}$ depot formulations; the physician's attitude towards depot, ${ }^{34}$ judgment of whether depot will be noticeably more effective than oral formulations, ${ }^{35}$ and experiences with prescribing depot; and the availability, cost, and resources needed to administer depot in each of the study countries and regions. ${ }^{36,37}$

The discrepancy between the physicians' perceptions of the patients' risk of nonadherence and the patients' assessment of their own adherence is of particular interest. Despite classifying their patients as being at risk of nonadherence, physicians may have elected for study inclusion those patients whose attitude toward taking antipsychotics was more positive (mean DAI-10 score at study entry was slightly positive) but whose behavior (whether they take the medication or not), ${ }^{38}$ or antipsychotic medication regimen, ${ }^{2,3}$ made them susceptible to nonadherence or treatment discontinuation. Few patients in the current study were considered at risk of nonadherence because of poor insight or a negative attitude to medication. Rather, most patients who were enrolled were considered at risk because of an inadequate response or intolerance to the primary antipsychotic medication. Most patients considered themselves to be adherent at study entry and this favorable self-perception increased during the study, possibly owing to selective attrition. Previous research has shown that patients tend to overestimate their medication adherence when reporting adherence. ${ }^{39}$ Given that patientreported adherence was measured using a subjective assessment scale, the patients' favorable self-perception of adherence in the current study may reflect their views and attitudes about adherence rather than actual adherence to their prescribed medication regimen. ${ }^{40}$ As patient adherence was not monitored by objective measures, the extent to which patients adhered to their medication regimen is not known, and even though the treatment discontinuation rate was low, patients may not have taken their medication as prescribed or were partially adherent to their antipsychotic medication regimen. ${ }^{41,42}$

Current findings need to be evaluated in the context of the study limitations. First, this study may not have enrolled those patients most susceptible to nonadherence, which in turn, may have contributed to the observed low treatment discontinuation rate. The proportion of patients (38.0\%) considered to be at risk of nonadherence that refused to 
participate in the current study suggests that enrolled patients were not a representative sample of the spectrum of nonadherent patients. Moreover, few patients were prescribed depot antipsychotics at study entry, which tends to be reserved for patients with a clear history of, or high risk for, nonadherence. ${ }^{11}$ Another possibility is that patients who were more engaged in their outpatient treatment may have been approached by physicians for enrollment in this study, as outpatients who are nonadherent to medication also tend to be nonadherent to their outpatient therapy program. ${ }^{43}$ Different sampling methods may need to be considered in future studies of this topic. Second, the five criteria used to identify risk of nonadherence in the current study may have resulted in physicians overlooking patients who were at risk because of other known predictors of nonadherence, such as recent illicit drug and alcohol abuse. ${ }^{19,20}$ Lastly, the generalization of the current findings to individual populations is challenging because of the distinct variability in antipsychotic prescribing practices across the study countries and regions, and differences in treatment discontinuation rates across countries and regions. ${ }^{44}$

\section{Conclusion}

In this 1-year naturalistic study, few patients discontinued their treatment for any reason even though these patients were considered at risk of nonadherence by their physicians. This finding was accompanied by significant improvements in the patients' clinical and functional status. Current results suggest the need for further research of patients with schizophrenia who are considered to be nonadherent to their antipsychotic medications and the need to understand the factors that may drive the marked discrepancy between the physicians' perception of their patients' adherence to the prescribed medication and the patients' assessment of their own adherence behaviors.

\section{Acknowledgments}

This study was sponsored by Eli Lilly and Company. Eli Lilly was involved in the study design, data collection, data analysis, and preparation of the manuscript. In compliance with the Uniform Requirements for Manuscripts, established by the International Committee of Medical Journal Editors, the sponsor of this study did not impose any impediment, directly or indirectly, on the publication of the study's results. The authors acknowledge statistical analysis assistance provided by George Zhao. The authors acknowledge the independent medical writing assistance provided by Julie Monk and Serina Stretton of ProScribe
Medical Communications (www.proscribe.com.au), funded from an unrestricted financial grant from Eli Lilly Australia. ProScribe's services complied with international guidelines for Good Publication Practice. The authors acknowledge Renee Granger for editorial support.

\section{Disclosure}

K Kelin, AJM Brnabic, J Karagianis, W Montgomery, W Ye, and $\mathrm{H}$ Ascher-Svanum are employees of Eli Lilly and Company. R Newton has received honoraria from AstraZeneca, Eli Lilly and Company, Janssen-Cilag Pty Limited, and Lundbeck Australia Pty Ltd. TJR Lambert has been on advisory boards and consulted for, and received honoraria and/or educational grants from, Eli Lilly and Company, Janssen-Cilag Pty Limited, Pfizer Inc, AstraZeneca, and Hospira, Inc. AJM Brnabic, J Karagianis, W Montgomery, and W Ye are shareholders in Eli Lilly and Company. K-P Chen, L Don and RI Escamilla have no conflicts of interest to disclose.

\section{References}

1. Canadian Psychiatric Association. Clinical practice guidelines. Treatment of schizophrenia. Can J Psychiatry. 2005;50(Suppl 1):7-57.

2. Haro JM, Novick D, Suarez D, Roca M. Antipsychotic treatment discontinuation in previously untreated patients with schizophrenia: 36-month results from the SOHO study. J Psychiatr Res. 2009;43: 265-273.

3. Lieberman JA, Stroup TS, McEvoy JP, et al. Effectiveness of antipsychotic drugs in patients with chronic schizophrenia. $N$ Engl $J$ Med. 2005;353:1209-1223.

4. Valenstein M, Copeland LA, Blow FC, et al. Pharmacy data identify poorly adherent patients with schizophrenia at increased risk for admission. Medical Care. 2002;40:630-639.

5. Weiden PJ, Kozma C, Grogg A, Locklear J. Partial compliance and risk of rehospitalization among California Medicaid patients with schizophrenia. Psychiatr Serv. 2004;55:886-891.

6. Svarstad BL, Shireman TI, Sweeney JK. Using drug claims data to assess the relationship of medication adherence with hospitalization and costs. Psychiatr Serv. 2001;52:805-811.

7. Liu-Seifert H, Adams DH, Kinon BJ. Discontinuation of treatment of schizophrenic patients is driven by poor symptom response: a pooled post-hoc analysis of four atypical antipsychotic drugs. BMC Med. 2005;3:21.

8. Ascher-Svanum H, Zhu B, Faries D, Landbloom R, Swartz M, Swanson J. Time to discontinuation of atypical versus typical antipsychotics in the naturalistic treatment of schizophrenia. BMC Psychiatry. 2006;6:8.

9. Dunayevich E, Ascher-Svanum H, Zhao F, et al. Longer time to antipsychotic treatment discontinuation for any cause is associated with better functional outcomes for patients with schizophrenia, schizophreniform disorder, or schizoaffective disorder. J Clin Psychiatry. 2007;68:1163-1171.

10. Corrigan PW, Liberman RP, Engel JD. From noncompliance to collaboration in the treatment of schizophrenia. Hosp Community Psychiatry. 1990;41:1203-1211.

11. Masand PS, Roca M, Turner MS, Kane JM. Partial adherence to antipsychotic medication impacts the course of illness in patients with schizophrenia: a review. Prim Care Companion J Clin Psychiatry. 2009;11:147-154. 
12. Stroup TS, McEvoy JP, Swartz MS, et al. The National Institute of Mental Health Clinical Antipsychotic Trials of Intervention Effectiveness (CATIE) project: schizophrenia trial design and protocol development. Schizophr Bull. 2003;29:15-31.

13. Chen L, McCombs JS, Park J. Duration of antipsychotic drug therapy in real-world practice: a comparison with CATIE trial results. Value Health. 2008; 11:487-496.

14. Haro JM, Novick D, Belger M, Jones PB. Antipsychotic type and correlates of antipsychotic treatment discontinuation in the outpatient treatment of schizophrenia. Eur Psychiatry. 2006;21:41-47.

15. Kilzieh N, Todd-Stenberg JA, Kennedy A, Wood AE, Tapp AM. Time to discontinuation and self-discontinuation of olanzapine and risperidone in patients with schizophrenia in a naturalistic outpatient setting. J Clin Psychopharmacol. 2008;28:74-77.

16. Mullins CD, Obeidat NA, Cuffel BJ, Naradzay J, Loebel AD. Risk of discontinuation of atypical antipsychotic agents in the treatment of schizophrenia. Schizophr Res. 2008;98:8-15.

17. Ascher-Svanum H, Zhu B, Faries DE, Lacro JP, Dolder CR, Peng X. Adherence and persistence to typical and atypical antipsychotics in the naturalistic treatment of patients with schizophrenia. Patient Prefer Adherence. 2008;2:67-77.

18. Dossenbach M, Arango-Dávila C, Silva Ibarra H, et al. Response and relapse in patients with schizophrenia treated with olanzapine, risperidone, quetiapine, or haloperidol: 12-month follow-up of the Intercontinental Schizophrenia Outpatient Health Outcomes (IC-SOHO) study. J Clin Psychiatry. 2005;66:1021-1030.

19. Ascher-Svanum H, Zhu B, Faries D, Lacro JP, Dolder CR. A prospective study of risk factors for nonadherence with antipsychotic medication in the treatment of schizophrenia. J Clin Psychiatry. 2006;67: 1114-1123.

20. Lacro JP, Dunn LB, Dolder CR, Leckband SG, Jeste DV. Prevalence of and risk factors for medication nonadherence in patients with schizophrenia: a comprehensive review of recent literature. J Clin Psychiatry. 2002;63:892-909.

21. Kelin K, Brnabic AJM, Newton R, et al. Baseline characteristics and initial treatment decisions for patients with schizophrenia at risk of treatment nonadherence. Pat Pref Adherence. 2010;4:301-311.

22. American Psychiatric Association (APA). Diagnostic and statistical manual of mental disorders (DSM-IV). Washington DC: American Psychiatric Association; 1994.

23. Guy W. ECDEU assessment manual for psychopharmacology. Rockville, MD: US Department of Health, Education, and Welfare, Public Health Service, Alcohol, Drug Abuse, and Mental Health Administration, National Institute of Mental Health, Psychopharmacology Research Branch, Division of Extramural Research Programs; 1976.

24. Hogan TP, Awad AG, Eastwood R. A self-report scale predictive of drug compliance in schizophrenics: reliability and discriminative validity. Psychol Med. 1983;13:177-183.

25. The EuroQol Group: EuroQol - a new facility for the measurement of health-related quality of life. Health Policy. 1990;16:199-208.

26. Ware JE Jr, Sherbourne CD. The MOS 36-item short-form health survey (SF-36). I. Conceptual framework and item selection. Med Care. 1992;30:473-483.

27. Dolan P. Modeling valuations for EuroQol health states. Med Care. 1997;35:1095-1108.

28. Karagianis J, Williams R, Davis L, et al. Antipsychotic switching: results from a one-year prospective, observational study of patients with schizophrenia. Curr Med Res Opin. 2009;25:2121-2132.

Patient Preference and Adherence

\section{Publish your work in this journal}

Patient Preference and Adherence is an international, peer-reviewed, open access journal focusing on the growing importance of patient preference and adherence throughout the therapeutic continuum. Patient satisfaction, acceptability, quality of life, compliance, persistence and their role in developing new therapeutic modalities and compounds to optimize
29. Serretti A, De Ronchi D, Lorenzi C, Berardi D. New antipsychotics and schizophrenia: a review on efficacy and side effects. Curr Med Chem. 2004;11:343-358.

30. Tiihonen J, Wahlbeck K, Lönnqvist J, et al. Effectiveness of antipsychotic treatments in a nationwide cohort of patients in community care after first hospitalisation due to schizophrenia and schizoaffective disorder: observational follow-up study. BMJ. 2006;333:224.

31. Zhu B, Ascher-Svanum H, Shi L, Faries D, Montgomery W, Marder SR. Time to discontinuation of depot and oral first-generation antipsychotics in the usual care of schizophrenia. Psychiatr Serv. 2008;59:315-317.

32. Heres S, Schmitz FS, Leucht S, Pajonk FG. The attitude of patients towards antipsychotic depot treatment. Int Clin Psychopharmacol. 2007; 22:275-282.

33. Patel MX, de Zoysa N, Bernadt M, David AS. A cross-sectional study of patients' perspectives on adherence to antipsychotic medication: depot versus oral. J Clin Psychiatry. 2008;69:1548-1556.

34. Heres S, Hamann J, Kissling W, Leucht S. Attitudes of psychiatrists toward antipsychotic depot medication. J Clin Psychiatry. 2006;67: 1948-1953.

35. Hamann J, Mendel R, Heres S, Leucht S, Kissling W. How much more effective do depot antipsychotics have to be compared to oral antipsychotics before they are prescribed? Eur Neuropsychopharmacol. 2010; 20:276-279

36. Chong MY, Tan CH, Fujii S, et al. Antipsychotic drug prescription for schizophrenia in East Asia: rationale for change. Psychiatry Clin Neurosci. 2004;58:61-67.

37. Yang YK, Tarn YH, Wang TY, et al. Pharmacoeconomic evaluation of schizophrenia in Taiwan: model comparison of long-acting risperidone versus olanzapine versus depot haloperidol based on estimated costs. Psychiatry Clin Neurosci. 2005;59:385-394.

38. Weiden PJ. Understanding and addressing adherence issues in schizophrenia: from theory to practice. J Clin Psychiatry. 2007;68(Suppl 14): 14-19.

39. Velligan DI, Lam YW, Glahn DC, et al. Defining and assessing adherence to oral antipsychotics: a review of the literature. Schizophr Bull. 2006;32:724-742

40. Barbui C, Kikkert M, Mazzi MA, et al. Comparison of patient and clinician perspectives in the assessment of antipsychotic medication adherence. Psychopathology. 2009;42:311-317.

41. Cooper D, Moisan J, Grégoire JP. Adherence to atypical antipsychotic treatment among newly treated patients: a population-based study in schizophrenia. J Clin Psychiatry. 2007;68:818-825.

42. Byerly M, Fisher R, Whatley K, et al. A comparison of electronic monitoring vs clinician rating of antipsychotic adherence in outpatients with schizophrenia. Psychiatry Res. 2005;133:129-133.

43. Nosé M, Barbui C, Tansella M. How often do patients with psychosis fail to adhere to treatment programmes? A systematic review. Psychol Med. 2003;33:1149-1160.

44. Bitter I, Treuer T, Dyachkova Y, Martenyi F, McBride M, Ungvari GS. Antipsychotic prescription patterns in outpatient settings: 24-month results from the Intercontinental Schizophrenia Outpatient Health Outcomes (IC-SOHO) study. Eur Neuropsychopharmacol. 2008;18: 170-180.

\section{Dovepress}

clinical outcomes for existing disease states are major areas of interest. This journal has been accepted for indexing on PubMed Central. The manuscript management system is completely online and includes a very quick and fair peer-review system. Visit http://www.dovepress.com/ testimonials.php to read real quotes from published authors. 